\title{
Tamoxifen for women at high risk of breast cancer
}

\author{
This article was published in the following Dove Press journal: \\ Breast Cancer:Targets and Therapy \\ 17 February 2014 \\ Number of times this article has been viewed
}

\author{
Safia A Nazarali \\ Steven A Narod \\ Women's College Research Institute, \\ Women's College Hospital, and \\ The University of Toronto, Toronto, \\ Ontario, Canada
}

\begin{abstract}
Tamoxifen has been used as a treatment for women who have been diagnosed with breast cancer for roughly four decades and has been approved as chemoprevention for over ten years. Although tamoxifen has been proven to be beneficial in preventing breast cancer in high-risk women, its use has not been widely embraced. To some extent, this is due to several of its side effects, including an increased risk of endometrial cancer and pulmonary embolism, but these serious side effects are rare. The risks and benefits of tamoxifen chemoprevention should be considered for each patient.
\end{abstract}

Keywords: tamoxifen, breast cancer, women, chemoprevention

\section{Introduction}

A large proportion of breast cancers express the estrogen receptor (ER), and the progression of these cancers is dependent on intact estrogen signaling. Treatment of ER-positive breast cancers usually includes a therapy that is designed to block estrogen signaling. Tamoxifen is an ER antagonist that has been used as a treatment for patients with breast cancer since the early $1970 \mathrm{~s} .{ }^{1}$ A collaborative meta-analysis conducted by the Early Breast Cancer Trialists Collaborative Group found that, for women with ER-positive breast cancer, 5 years of tamoxifen therapy reduces the annual breast cancer death rate by $31 \%{ }^{2}$ In addition, tamoxifen lowers the risk of developing a contralateral breast cancer by as much as one half. ${ }^{3}$ This observation led scientists to believe that tamoxifen might be effective as a chemopreventive agent in healthy women who are at increased risk of developing the disease. ${ }^{1}$ Several randomized trials have been conducted to test this hypothesis and these support the use of tamoxifen as a chemopreventive agent in high-risk women. As a result, the National Institute for Health and Care Excellence (NICE) in the UK recently updated its cancer prevention guidelines for women who are at high risk of developing the disease. ${ }^{4}$ They recommend that women who have a strong family history of breast cancer but who have not been diagnosed with the disease be offered 5 years of chemoprevention with tamoxifen or raloxifene. ${ }^{4}$ NICE also advocates for more women to be tested for genetic mutations that are known to cause cancer (BRCA1 and $B R C A 2)$ and proposed more inclusive criteria for genetic testing. ${ }^{4}$ This policy update in the UK is due in part to the greater acceptance of the value of chemoprevention in high-risk women, including those who carry a $B R C A$ mutation.

It is important to consider the risks associated with tamoxifen use and to determine the risks and benefits for each group of women. Four groups of women who
Women's College Research Institute 790 Bay Street, Toronto, Ontario, Canada M5GIN8

Email steven.narod@wchospital.ca 
are at high risk of developing breast cancer are discussed here, including women with a family history of the disease, women with a BRCA1 or BRCA2 mutation, women with atypical hyperplasia in their breast tissue, and women with high density mammograms.

\section{Tamoxifen metabolism}

Estrogen binds to hormone receptors and causes increased cell proliferation in the breast tissue. Tamoxifen blocks estrogen by binding to these receptors, and is therefore classified as an ER antagonist with respect to breast tissue. Tamoxifen itself has a weak affinity for the ER. ${ }^{5}$ It is converted to active and inactive metabolites, which have a higher affinity for such receptors. ${ }^{5} \mathrm{~N}$-dimethyl-tamoxifen is the primary metabolite, which is subsequently metabolized into $\alpha$-hydroxy-tamoxifen, $\mathrm{N}$-didesmethyl-tamoxifen, and 4-hydroxy-N-desmethyltamoxifen (also known as endoxifen). ${ }^{5}$ Tamoxifen is also converted to 4-hydroxy-tamoxifen; a reaction catalyzed by many enzymes, including cytochrome P450 2D6, as can be seen in Figure $1 .^{5}$ Endoxifen and 4-hydroxy-tamoxifen have at least a 10-fold higher affinity for ERs than tamoxifen. ${ }^{5}$ In fact, in patients receiving tamoxifen therapy, endoxifen is found in serum concentrations 6-12-fold higher than 4-hydroxytamoxifen. ${ }^{5}$ Thus, endoxifen is likely the most important metabolite required for tamoxifen activity. ${ }^{5}$

\section{High-risk groups}

The National Surgical Adjuvant Breast and Bowel Project (NSABP) Breast Cancer Prevention Trial was designed to observe the effectiveness of tamoxifen as a chemopreventive agent in high-risk women. Eligibility was based on risk level and required that the participants have either a history of lobular carcinoma in situ, be aged 60 years or older, or have $\mathrm{a} \geq 1.66 \%$ risk of breast cancer over the next 5 years. A total of 13,338 women at high risk of breast cancer were randomized to receive either oral tamoxifen or placebo for 5 years. Tamoxifen use resulted in a $43 \%$ reduction in invasive breast cancer (hazards ratio [HR] 0.57, 95\% confidence interval [CI]

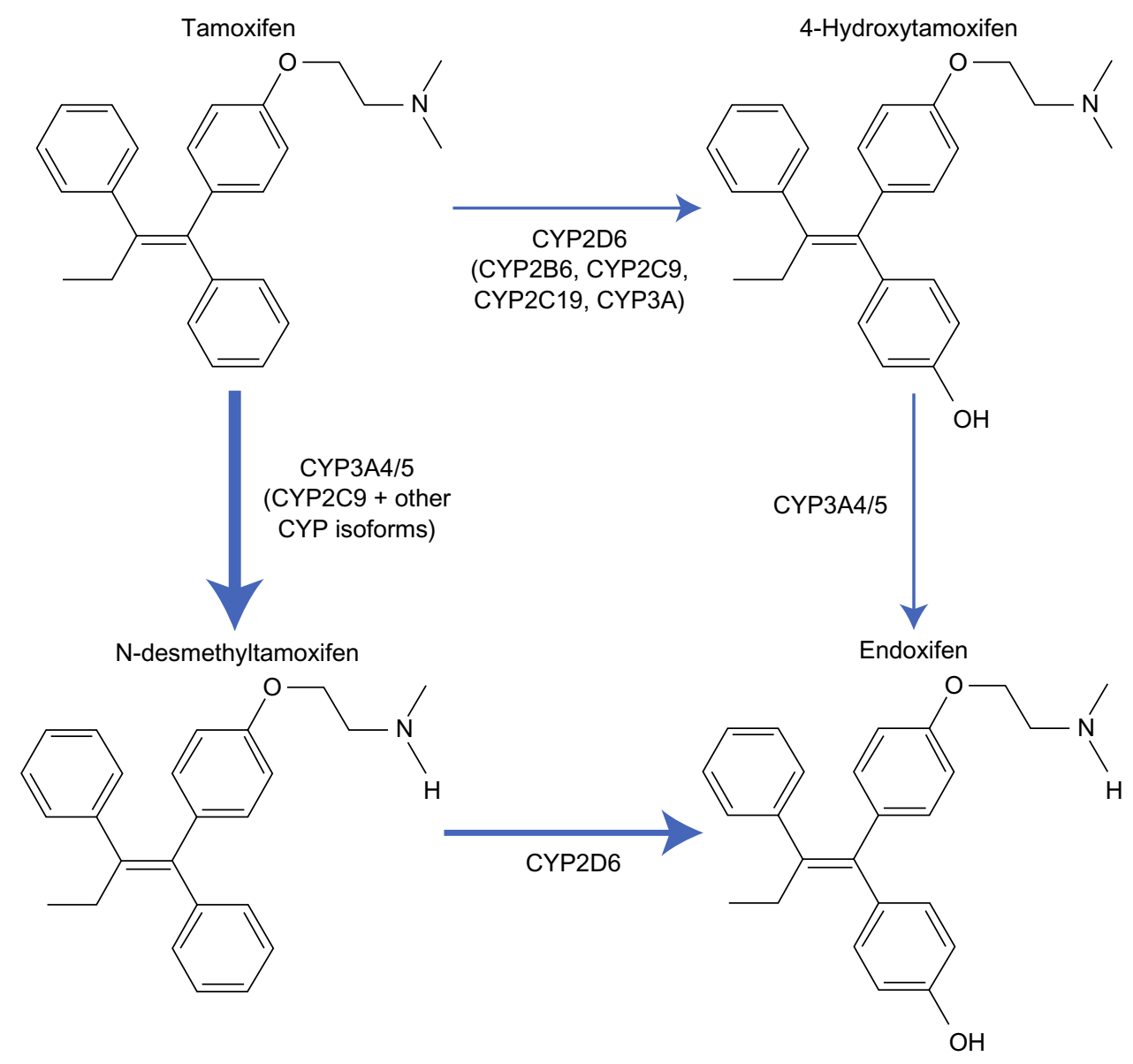

Figure I Overview of tamoxifen metabolism pathway and the main enzymes involved. Thickness of the arrow demonstrates the relative contribution of each pathway. Note: Adapted from Springer and Current Oncology Reports, 12,2010,7-15, CYP2D6 polymorphisms and tamoxifen metabolism: clinical relevance, Higgins MJ, Stearns V, figure number I, with kind permission from Springer Science and Business Media.

Abbreviations: CYP, cytochrome P450; OH, hydroxide. 
0.46-0.70). ${ }^{6}$ The reduction was only seen for ER-positive cancers (HR 0.38, 95\% CI 0.28-0.50). The magnitude of risk reduction varied by subgroup: women with a history of atypical hyperplasia experienced a risk reduction of $75 \%$ (HR $0.25,95 \%$ CI $0.10-0.52){ }^{6}$ The study investigators planned to evaluate the protective effect separately for white and black women but there were too few black women enrolled in the study to generate an estimate for this subgroup.

\section{Family history}

Approximately $8 \%$ of breast cancer patients have an affected first-degree relative. ${ }^{7}$ Women with a strong family history of breast cancer are a natural group to use to study chemopreventive agents for several reasons. These women tend to be motivated to participate as they have an increased risk of developing the disease and personal experience of breast cancer in a family member. Second, studies of high-risk women are more efficient with regards to cost. If there are side effects associated with chemoprevention, using women at high risk will optimize the risk/benefit ratio. The three main studies that have been conducted to study the effect of chemopreventive tamoxifen in women with a strong family history of breast cancer are The Royal Marsden Trial, The International Breast Cancer Intervention Study, and the Milan study.

The Royal Marsden Trial accrued 2,494 women between the ages of 30 and 70 years who had an increased risk of developing breast cancer due to a strong family history. ${ }^{8}$ Participants were randomly assigned to either tamoxifen $20 \mathrm{mg} /$ day or a placebo for 8 years and were followed for an additional 8 years. After patients were followed for up to 16 years, the risk of ER-positive invasive breast cancer was significantly lower in the tamoxifen group than in the placebo group (HR 0.61, 95\% CI 0.43-0.86, $P=0.005$ ) ${ }^{8}$ the risk of ER-negative breast cancer was not significantly lower in the tamoxifen group (HR 1.4, 95\% CI 0.7-2.6, $P=0.3$ ). The HR for any invasive cancer was 0.78 (95\% CI $0.58-1.04)$ and was not statistically significant $(P=0.1)$.

In 2008, the International Breast Cancer Intervention Study randomly assigned 7,145 women aged $35-70$ years who were at increased risk of breast cancer to receive tamoxifen 20 $\mathrm{mg}$ /day or placebo for 5 years. ${ }^{9}$ Of these women, 3,433 had a first-degree relative with early-onset breast cancer and 4,410 had two or more first-degree or second-degree relatives with breast cancer. ${ }^{9}$ After a median follow-up of 96 months, 142 women in the tamoxifen group were diagnosed with breast cancer compared with 195 women in the placebo group (HR $0.73,95 \%$ CI $0.58-0.91, P=0.004) .{ }^{9}$ The reduction was limited to ER-positive cancers (HR 0.66, 95\% CI 0.50-0.87).
A third study was initiated in Milan, Italy, in 1992 to assess the benefit of tamoxifen as a chemopreventive agent in Italian women with a strong family history of breast cancer. Because of the concern for endometrial cancer, only women who had a hysterectomy were included. Women were randomized to receive tamoxifen $20 \mathrm{mg}$ /day or placebo for 5 years. After 11 years of follow-up, a total of 136 women had developed breast cancer (74 in the placebo group and 62 in the tamoxifen group). ${ }^{10}$ The overall association with tamoxifen was not significant (HR 0.84, 95\% CI 0.60-1.17), nor was there a significant reduction in the risk of ER-positive cancers (HR 0.77, 95\% CI 0.51-1.16).

\section{BRCA mutations}

The lifetime risk of a woman developing breast cancer is about $70 \%$ for a $B R C A 1$ mutation carrier and $60 \%$ for a BRCA2 mutation carrier. ${ }^{11}$ Women who undergo genetic testing and are found to be positive for a mutation have several options for risk management, including prophylactic surgery (mastectomy and/or oophorectomy) or chemopreventive drugs such as tamoxifen.

The Hereditary Breast Cancer Clinical Study Group conducted a case-control study matching 285 women with bilateral breast cancer and known $B R C A$ mutation to 751 $B R C A$ mutation carriers with unilateral breast cancer. ${ }^{12}$ The results of this study showed that tamoxifen use was associated with a $50 \%$ reduction in the risk of developing a contralateral breast cancer in mutation carriers (odds ratio $0.50,95 \% \mathrm{CI}$ $0.30-0.85) .{ }^{12}$ Researchers observed a benefit of tamoxifen for up to 4 years of use. ${ }^{12}$

Metcalfe et al conducted a historical cohort study observing the protective effect of tamoxifen on women with a $B R C A 1$ or BRCA2 mutation. Recruited were 491 women with stage I or stage II breast cancer, for whom a $B R C A 1$ or $B R C A 2$ mutation was identified in the family. The results showed a moderate decrease in the risk of contralateral breast cancer associated with tamoxifen use (HR 0.59, 95\% CI 0.35-1.01, $P=0.05) .{ }^{13}$ A greater protective effect was observed for $B R C A 1$ carriers (HR 0.57) than for $B R C A 2$ carriers (HR 0.73). ${ }^{13}$ However, this difference was not statistically significant. ${ }^{13}$

A recent study was conducted by Phillips et al to determine if adjuvant tamoxifen therapy is associated with a reduced risk of contralateral breast cancer in women with a BRCA1 or BRCA2 mutation. ${ }^{14}$ In total, 383 of 1,583 BRCA1 mutation carriers and 454 of 881 BRCA2 mutation carriers took tamoxifen after being diagnosed with breast cancer. ${ }^{14}$ Analysis of combined historical and prospective data showed 
a significant reduction in the risk of contralateral breast cancer with the use of tamoxifen in $B R C A 1$ mutation carriers (HR 0.38, $P<0.001$ ) and in $B R C A 2$ mutation carriers (HR 0.33, $P<0.001) .{ }^{14}$

The findings of Metcalfe et al and Phillips et al indicate that adjuvant tamoxifen therapy reduces the risk of contralateral breast cancer in women who have been diagnosed with breast cancer and are $B R C A 1$ or $B R C A 2$ mutation carriers. However, to date, there are no prospective studies specifically designed to study chemoprevention for primary breast cancer in BRCA1 or BRCA2 carriers. Very few mutation carriers choose to use tamoxifen due to the perceived side effects associated with treatment. A small prospective study of chemoprevention for primary breast cancer in $B R C A$ mutation carriers was conducted in 2001 . King et al $^{8}$ tested 288 women who developed breast cancer in the randomized NSABP Breast Cancer Prevention Trial of BRCA mutations. Of these 288 women, eight were found to have a $B R C A 1$ mutation and eleven were found to have a $B R C A 2$ mutation. ${ }^{15}$ Tamoxifen reduced the breast cancer risk by $62 \%$ in $B R C A 2$ carriers, ${ }^{15}$ but did not reduce the risk of breast cancer in healthy women with a BRCA1 mutation. ${ }^{15}$

In summary, the results of large studies in BRCA1 and $B R C A 2$ carriers differ substantially from those in noncarriers, in that among mutation carriers the protective effect of tamoxifen does not appear to be limited to prevention of ER-positive cancers. This is a consistent observation across the four randomized trials conducted in noncarriers (Table 1), including those in which most study subjects had a strong family history of breast cancer.

\section{Atypical hyperplasia}

Atypical hyperplasia is the accumulation of abnormal cells in breast tissue due to an increased proliferation of breast tissue cells. It can occur in one or both breasts and is usually found at breast biopsy. It is classified as a benign condition, but it increases the risk of subsequent breast cancer by 4-5 times. ${ }^{16}$ The NSABP P-1 study reported a $75 \%$ reduction in the incidence of breast cancer in women with atypical hyperplasia who received tamoxifen rather than placebo. ${ }^{6}$ Shortly after closing of the NSABP P-1 trial, researchers continued to follow women whose breast tissue showed atypical hyperplasia or lobular carcinoma in situ. ${ }^{6}$ The incidence of invasive breast cancer in 1,000 women with atypical hyperplasia was 10.1 in the placebo group, compared with 1.4 in the tamoxifen group (relative risk [RR] 0.14, 95\% CI 0.03-0.47). ${ }^{6}$ This estimates a 5 -year breast cancer rate of approximately $5.0 \%{ }^{6}$ Similarly, the incidence of invasive breast cancer per 1,000 women with lobular carcinoma in situ was 13.0 in the placebo group compared with 5.7 in the tamoxifen group (RR 0.44, 95\% CI 0.16-1.06). ${ }^{6}$ These findings confirm that tamoxifen significantly reduces the risk of invasive breast cancer in women with atypical hyperplasia or lobular carcinoma in situ.

Another recent trial studying the effect of tamoxifen in women with atypical hyperplasia yielded a similar result. Coopey et al reviewed the breast pathology reports of 42,950 women from 1987 to 2010 in order to determine the benefit of chemoprevention in patients with atypical breast lesions. ${ }^{17}$ They identified 2,459 women with atypical breast lesions without prior or concurrent breast cancer, and of these

Table I Summary of major randomized trials of tamoxifen prevention

\begin{tabular}{|c|c|c|c|c|c|}
\hline \multirow[t]{2}{*}{ Study (reference) } & \multirow{2}{*}{$\begin{array}{l}\text { Study } \\
\text { subjects (n) }\end{array}$} & \multirow{2}{*}{$\begin{array}{l}\text { Subject } \\
\text { age, years }\end{array}$} & \multicolumn{2}{|c|}{ Events (n) } & \multirow[t]{2}{*}{ RR (95\% Cl) } \\
\hline & & & Placebo & Tamoxifen & \\
\hline$\overline{N S A B P}{ }^{6}$ & 13,388 & $35-80$ & & & \\
\hline All invasive & & & 250 & 145 & $0.57(0.46-0.70)$ \\
\hline ER-positive & & & 182 & 70 & $0.38(0.28-0.50)$ \\
\hline ER-negative & & & 42 & 56 & $1.31(0.86-2.01)$ \\
\hline Royal Marsden Trial ${ }^{8}$ & 2,494 & $30-70$ & & & \\
\hline All invasive & & & 104 & 82 & $0.78(0.58-1.04)$ \\
\hline ER-positive & & & 86 & 53 & $0.61(0.43-0.86)$ \\
\hline ER-negative & & & 17 & 24 & $1.4(0.7-2.6)$ \\
\hline International Breast & 7,145 & $35-70$ & 195 & 142 & $0.73(0.58-0.91)$ \\
\hline \multicolumn{6}{|l|}{ Cancer Intervention } \\
\hline \multicolumn{6}{|l|}{ Study ${ }^{9}$} \\
\hline ER-positive & & & 132 & 35 & $0.66(0.50-0.87)$ \\
\hline ER-negative & & & 35 & & $1.00(0.53-1.87)$ \\
\hline Milan study ${ }^{10}$ & 5,408 & $35-70$ & 74 & 62 & $0.84(0.60-1.17)$ \\
\hline ER-positive & & & 52 & 40 & $0.77(0.5 \mathrm{I}-\mathrm{I} . \mathrm{I} 6)$ \\
\hline ER-negative & & & 19 & 21 & $1.10(0.59-2.05)$ \\
\hline
\end{tabular}

Abbreviations: $\mathrm{Cl}$, confidence interval; ER, estrogen receptor; RR, relative risk; NSABP, National Surgical Adjuvant Breast and Bowel Project. 
women, 466 were treated with chemoprevention, primarily with tamoxifen, and 1,472 were not. ${ }^{17}$ The results showed a significant decrease in risk of breast cancer with tamoxifen $(P<0.001) .{ }^{17}$ Women over the age of 50 years with atypia were found to have a significant reduction in breast cancer risk with chemoprevention (HR 0.34, $P=0.001) .{ }^{17}$ For women under the age of 50 years, the reduction was smaller and not statistically significant (HR $0.70 ; P=0.33$ ). ${ }^{17}$ These findings suggest that tamoxifen therapy is particularly beneficial in reducing the risk of breast cancer in patients with atypical breast lesions.

\section{High-density mammograms}

Extensive dense breast tissue visible on a mammogram is a strong risk factor for breast cancer. ${ }^{18}$ High-density mammograms are an indicator of a higher proportion of glandular and connective tissue in the breast relative to fat. Women with high-density mammograms have a cancer risk up to six times that of women with low-density mammograms. ${ }^{19}$

Studies have shown that tamoxifen reduces mammographic breast density. The 2008 International Breast Cancer Intervention Study showed that the mean reduction in mammographic breast density amongst women in the tamoxifen arm was $8.2 \%$, and $48 \%$ of women had a $\geq 10 \%$ reduction in breast density at their $12-18$-month mammogram. ${ }^{18}$ In comparison, the mean density reduction amongst women in the placebo group was $3.8 \%$, and only $26 \%$ of these women experienced a $\geq 10 \%$ reduction in breast density at their 12-18-month mammogram. ${ }^{18}$ In comparison with women in the placebo group, those who took tamoxifen and experienced $\mathrm{a} \geq 10 \%$ reduction in breast density had a $\mathrm{RR}$ reduction in breast cancer risk of $63 \%$ (odds ratio $0.37,95 \%$ CI $0.20-0.69$, $P=0.002) .{ }^{18}$ Women who were taking hormone replacement therapy did not experience a reduction in breast cancer risk with tamoxifen. ${ }^{18}$

In 2012, Chen et al conducted a study using threedimensional magnetic resonance imaging to examine the effect of tamoxifen therapy on breast density. The results showed that tamoxifen therapy causes a significant reduction in breast density, and this reduction is positively correlated with treatment duration and baseline breast density. ${ }^{19}$ The findings from these studies suggest that tamoxifen therapy is beneficial in reducing breast density, and reduces the risk of invasive breast cancer in this high-risk group. Nevertheless, more research is needed to confirm that a reduction in breast density due to tamoxifen is accompanied by a commensurate reduction in breast cancer risk and that tamoxifen is ineffective in the absence of a reduction in breast density.

\section{Risks of tamoxifen use}

Although tamoxifen has shown clear benefit in the prevention of breast cancer in high-risk women, many patients are apprehensive about taking this drug due to its side effect profile. There are several risks associated with use of tamoxifen, which may be attributed to its estrogenic activity in human endometrial tissue. ${ }^{1}$ During the NSABP P-1 trial, 51 invasive endometrial cancers occurred in the active phase of treatment, ie, 15 in the placebo arm and 36 in the tamoxifen $\operatorname{arm}(\mathrm{RR} 1.13,95 \%$ CI 0.44-2.93, $P=0.003) .{ }^{20}$ Age has been shown to predict the risk of developing invasive endometrial cancer. The NSABP trial showed that in women $\geq 50$ years of age, the RR of developing endometrial cancer was 3.86 (95\% CI 1.69-8.86), whereas in women $<50$ years of age, the RR of endometrial cancer was 1.13 (95\% CI 0.44-2.93). ${ }^{20}$ The NSABP trial also found that the use of tamoxifen in breast cancer patients resulted in an increased risk of 7.5-fold for endometrial tumors and a 1.4-5-fold increased risk of endometrial cancer in healthy women without breast cancer who were taking tamoxifen. ${ }^{21}$

Tamoxifen has also been shown to increase the incidence of deep vein thrombosis, pulmonary embolism, and cataracts. $^{1,21}$ Both deep vein thrombosis and pulmonary embolism pose significant health risks, and can lead to heart attacks, ischemic stroke, and death. During the NSABP-P1 trial, 57 women reported a deep vein thrombosis; 22 in the placebo arm and 35 in the tamoxifen arm (RR 1.60, 95\% CI 0.94-2.72, $P=0.1) .{ }^{20}$ Twenty-four pulmonary emboli were reported, ie, six in the placebo arm and 18 in the tamoxifen arm (RR 3.01, 95\% CI 1.20-1.58, $P=0.02) .{ }^{20}$ The increase in the annual rate of pulmonary embolism was not statistically significant in women $<50$ years of age $(P=0.4)$ but was significant in women aged $>50$ years $(P=0.01)$, suggesting that age is a factor when determining the risk of pulmonary emboli in these patients. ${ }^{20}$

\section{Attitudes of women and physicians towards use of tamoxifen}

Although tamoxifen has been used as a treatment for breast cancer since the early 1970 s, there is only limited enthusiasm for the drug in the preventive setting. The results of a multiethnic study of breast cancer risk reduction therapies indicated that even though $54 \%$ of women have heard of tamoxifen, only $4 \%$ have discussed it with their physicians. ${ }^{22}$

The recommended course of tamoxifen for prevention is 5 years; however, many women stop earlier than this. Land et al conducted a study to assess tamoxifen adherence in the NSABP-P1 trial. They found that $75 \%$ of study participants 
continued to take the recommended dose of the drug after one month, but only $41 \%$ of participants were still taking the recommended dose after 36 months. ${ }^{23}$ When asked why they chose to discontinue tamoxifen, most participants reported adverse effects. ${ }^{23}$ The study also found that participants who were 60 years or older were more likely to complete treatment than women younger than 60 years. ${ }^{23}$ Thus, despite the benefits associated with tamoxifen therapy for breast cancer prevention, widespread knowledge and adherence to such use of the drug is inadequate.

Armstrong et al conducted a study to assess factors affecting the prescription of tamoxifen as a chemopreventive agent among primary care physicians. Researchers mailed a survey to 350 primary care physicians, including those specializing in family practice, general internal medicine, and obstetrics and gynecology. ${ }^{24}$ The purpose of the survey was to collect information regarding prescription of tamoxifen, intention to prescribe tamoxifen in certain situations, and factors affecting willingness to prescribe the drug to women at high risk of breast cancer. The study found that $27.4 \%$ of the 350 physicians reported having prescribed tamoxifen as a chemopreventive agent for breast cancer at least once in the past year. ${ }^{24}$ The likelihood of prescribing tamoxifen was associated with the physician having a family member who had been diagnosed with breast cancer, the belief that the benefits of tamoxifen outweighed the risks, eligibility that was easy to determine, and patients who asked for more information about the drug. ${ }^{24}$ Thus, it is evident that few physicians prescribe tamoxifen as a chemopreventive agent to women who are at high risk of breast cancer, and their decision to prescribe the drug is affected by their perception of the effectiveness of the drug, their personal experience with breast cancer, patient interest, and their ability to determine the eligibility of their patients.

\section{Possible alternatives}

\section{Second-generation drugs}

Second-generation drugs, such as raloxifene and exemestane, may be alternatives to tamoxifen, in particular if they are equally or more effective and/or have fewer side effects. One study showed that after 4 years of treatment, raloxifene reduced the risk of invasive ER-positive breast cancer by $84 \% .^{1}$ In addition, raloxifene appears to be less estrogenic to the endometrium than tamoxifen. ${ }^{1}$ However, it may not be as effective; in 2010, Vogel et al assessed the results of the NSABP Study of Tamoxifen and Raloxifene (STAR) and found that the rate of invasive breast cancer was approximately $24 \%$ higher in patients taking raloxifene than in those taking tamoxifen (RR 1.24, 95\% CI 1.05-1.47). ${ }^{25}$ It was also found that the number of noninvasive breast cancer cases in the raloxifene group was twice that in the tamoxifen group (RR 2.00). ${ }^{25}$ Although tamoxifen is more effective in preventing both invasive and noninvasive breast cancer in high-risk women, raloxifene has been found to have a more favorable side effect profile. The results of this study showed that the raloxifene group had a significantly lower risk of developing uterine cancer (RR $0.55,95 \%$ CI $0.36-0.83, P=0.003$ ) and uterine hyperplasia (RR $0.19,95 \%$ CI $0.12-0.29$ ) than the tamoxifen group. ${ }^{25}$ In addition, the incidence of thromboembolic disease was considerably lower in the raloxifene group (RR 0.75, 95\% CI 0.60-0.93). ${ }^{25}$

Visvanathan et al conducted a meta-analysis to compare several different pharmacologic interventions for reduction of the risk of breast cancer. Their analysis confirmed that raloxifene results in fewer and less severe side effects than tamoxifen. Raloxifene was found to be associated with a lower risk of thromboembolic disease, uterine cancer, benign uterine hyperplasia, and cataracts. ${ }^{26}$ It was also associated with a lower incidence of ovarian cysts, endometrial polyps, hot flashes, and vaginal bleeding. ${ }^{26}$ It is clear that both raloxifene and tamoxifen are beneficial in preventing invasive breast cancer in high-risk women (with a risk reduction of $38 \%$ and $50 \%$, respectively). ${ }^{25}$

Exemestane has also been considered for breast cancer prevention in healthy, high-risk women. Exemestane is an aromatase inhibitor that binds to and inhibits the enzyme aromatase, leading to a reduction in estrogen levels. A recent study showed that exemestane resulted in a $65 \%$ relative reduction in the annual incidence of invasive breast cancer compared with placebo and was associated with no serious toxic effects after 3 years of use. ${ }^{27}$ However, more women in the exemestane group than in the tamoxifen group self-reported that their menopause-related vasomotor and sexual symptoms had worsened. ${ }^{27}$ Since exemestane depletes estrogen completely, menopausal symptoms may be more severe and loss of bone mineral density may cause bone pain. ${ }^{27}$ More severe side effects associated with exemestane include hypertension, hyperlipidemia, and cardiac failure. ${ }^{28}$

\section{Low-dose tamoxifen}

Scientists have suggested that administering lower doses of tamoxifen may achieve the benefit but reduce the side effects associated with the drug. Data from a recent animal study indicated that decreasing the tamoxifen dose to the human equivalent of $1 \mathrm{mg}$ per day does not reduce its antitumor 
effect in breast tissue, since this effect reaches a plateau once the ERs are saturated. ${ }^{29}$

Decensi et al compared the effects of different doses of tamoxifen on several biomarkers that reflect the activity of tamoxifen on target tissues. These biomarkers included lipid and insulin-like growth factor levels, as well as tissue biomarkers such as Ki-67. ${ }^{30}$ They studied 127 healthy women aged 35-70 years. ${ }^{30}$ Participants were randomly assigned to a placebo, tamoxifen $20 \mathrm{mg}$ /day, tamoxifen $10 \mathrm{mg} /$ day, or tamoxifen $10 \mathrm{mg}$ on alternate days. ${ }^{30} \mathrm{~A}$ reduction in the dose of tamoxifen by up to $75 \%$ of the conventional dose had no impact on the activity of the drug on these biomarkers. ${ }^{30}$ This observation has not been confirmed in a clinical setting. Research on this topic should be considered a priority.

Studies have been conducted to observe the effectiveness of combining low-dose tamoxifen with other agents to reduce the risk of breast cancer in healthy but high-risk women. However, these studies have shown that such combinations do not increase the efficacy of the drug. One study showed that the combination of low-dose tamoxifen and fenretinide did not reduce neoplastic breast events compared with placebo, whereas both single agents showed significant reduction of breast neoplasms. ${ }^{31}$ Another study found that a combination of tamoxifen and aromatase inhibitors results in less efficacy than tamoxifen alone. ${ }^{31}$ Use of tamoxifen alone is more effective than the combination of tamoxifen with other agents, as demonstrated in studies to date.

\section{Short-duration tamoxifen}

While tamoxifen has been shown to reduce the mortality rate in women with invasive breast cancer and to decrease the incidence of breast cancer in healthy, high-risk women, the optimal duration of tamoxifen therapy in the preventive setting remains controversial. The recommended dose and duration for prevention was based on studies of adjuvant tamoxifen and the main endpoint was cancer recurrence. A study was conducted by Alkner et al in 2009 using 564 premenopausal women with stage II breast cancers to analyze the effect of adjuvant tamoxifen therapy for a duration of 2 years. ${ }^{32}$ The results showed a significant reduction of contralateral breast cancer in premenopausal women with invasive breast cancer (HR 0.5, $P=0.02$ ). ${ }^{32}$ The idea that short-duration tamoxifen is beneficial in reducing the risk of developing contralateral breast cancer has led some scientists to believe that 2 years of tamoxifen therapy may be sufficient to prevent invasive breast cancer in healthy, high-risk women. Further research is required to confirm the benefit of short-duration tamoxifen for chemoprevention.

\section{Topical tamoxifen}

Scientists have suggested the use of topical tamoxifen as an alternative to oral administration. When delivered transdermally, a drug has longer retention in local tissue. ${ }^{29}$ Tamoxifen requires hepatic activation, so its more active metabolites should be used when it is administered transdermally. The tamoxifen metabolite 4-OHT can be concentrated directly in breast tissue..$^{29}$ One study showed that the maximal concentration of both tamoxifen and 4-OHT in breast tissue was reached 24 hours after topical application, with 4-OHT being retained in breast tissue for a much longer period than tamoxifen..$^{29}$ Rouanet et $\mathrm{al}^{33}$ conducted a study which found that when $1 \mathrm{mg}$ or $2 \mathrm{mg}$ of 4-OHT was applied to breast skin daily, sufficient concentrations of 4-OHT were reached in the breast tissue in order to achieve the same degree of inhibition of tumor cells as seen with $20 \mathrm{mg}$ /day of oral tamoxifen. ${ }^{29}$ The benefit of transdermal 4-OHT is that it results in much lower plasma levels compared with oral tamoxifen, possibly reducing toxicity, thus resulting in fewer side effects. ${ }^{29}$

\section{Conclusion}

Tamoxifen has been shown to be an effective chemopreventive agent for women who are at high risk of developing breast cancer. However, the risk/benefit ratio must be assessed for each woman. Women who would benefit from chemopreventive tamoxifen include those with a BRCA1 or BRCA2 mutation, atypical hyperplasia, and/or a high-density mammogram. Women with a strong family history of breast cancer but with no BRCA mutation are candidates for tamoxifen but are often reluctant to take it due to perceived side effects. Possible alternatives include raloxifene, low-dose tamoxifen, short-term tamoxifen, and topical tamoxifen. These alternatives may minimize toxicity while retaining activity. Further studies are needed to evaluate the effectiveness of such alternatives.

\section{Disclosure}

The authors report no conflicts of interest in this work.

\section{References}

1. Brown K. Breast cancer chemoprevention: risk-benefit effects of the anti-oestrogen tamoxifen. Expert Opin Drug Saf. 2002;1:253-267.

2. Early Breast Cancer Trialists Collaborative Group. Effects of chemotherapy and hormonal therapy for early breast cancer on recurrence and 15-year survival: an overview of the randomised trials. Lancet. 2005;365: 1687-1717.

3. Early Breast Cancer Trialists Collaborative Group. Tamoxifen for early breast cancer: an overview of the randomized trials. Lancet. 1998;351: 1451-1467. 
4. National Institute for Health and Care Excellence. Major shift in breast cancer care on horizon as NICE recommends preventative drugs for 'at-risk' women. Available from: http://www.nice.org.uk/newsroom/ pressreleases/NICERecommendsBreastCancerdrugs.jsp. Accessed November 27, 2013.

5. Higgins MJ, Stearns V. CYP2D6 polymorphisms and tamoxifen metabolism: clinical relevance. Curr Oncol Rep. 2010;12:7-15.

6. Fisher B, Costantino JP, Wickerham DL, et al. Tamoxifen for prevention of breast cancer: current status of the National Surgical Adjuvant Breast and Bowel Project P-1 Study. J Natl Cancer Inst. 2005;97: 1652-1662.

7. Nayfield SG, Karp JE, Ford LG, et al. Potential role of tamoxifen in prevention of breast cancer. J Natl Cancer Inst. 1991;83:1450-1459.

8. Powles TJ, Ashley S, Tidy A, et al. Twenty-year follow-up of the Royal Marsden randomized, double-blinded tamoxifen breast cancer prevention trial. J Natl Cancer Inst. 2007;99:283-290.

9. Cuzick J, Forbes JF, Sestak I, et al. Long-term results of tamoxifen prophylaxis for breast cancer - 96-month follow-up of the randomized IBIS-I trial. J Natl Cancer Inst. 2007;99:272-282.

10. Veronesi U, Maisonneuve P, Rotmensz N, et al. Tamoxifen for the prevention of breast cancer: late results of the Italian Randomized Tamoxifen Prevention Trial Among Women With Hysterectomy. J Natl Cancer Inst. 2007;99:727-737.

11. Narod SA. BRCA mutations in the management of breast cancer: the state of the art. Nat Rev Clin Oncol. 2010;7:702-707.

12. Gronwald J, Tung N, Foulkes WD, et al; Hereditary Breast Cancer Clinical Study Group. Tamoxifen and contralateral breast cancer in BRCA1 and BRCA2 carriers: an update. Int J Cancer. 2006;118:2281-2284.

13. Metcalfe K, Lynch H, Ghadirian P, et al. Contralateral breast cancer in BRCA1 and BRCA2 mutation carriers. J Clin Oncol. 2004;22: 2328-2335.

14. Phillips KA, Milne R, Rookus M, et al. Tamoxifen and risk of contralateral breast cancer for BRCA1 and BRCA2 mutation carriers. J Clin Oncol. 2013;31:3091-3099.

15. King MC, Wieand S, Hale K, et al; National Surgical Adjuvant Breast and Bowel Project. Tamoxifen and breast cancer incidence among women with inherited mutations in BRCA1 and BRCA2. JAMA. 2001;286:2251-2256.

16. Whiffen A, El-Tamer M, Taback B, Feldman S, Joseph KA. Predictors of breast cancer development in women with atypical ductal hyperplasia and atypical lobular hyperplasia. Ann Surg Oncol. 2011;18:463-467.

17. Coopey SB, Mazzola E, Buckley JM, et al. The role of chemoprevention in modifying the risk of breast cancer in women with atypical breast lesions. Breast Cancer Res Treat. 2012;136:627-633.

18. Cuzick J, Warwick J, Pinney E, et al. Tamoxifen-induced reduction in mammographic density and breast cancer risk reduction: a nested case-control study. J Natl Cancer Inst. 2011;103:744-752.

19. Chen J-H, Change Y-C, Chang D, et al. Reduction of breast density following tamoxifen treatment evaluated by 3-D MRI: preliminary study. Magn Reson Imaging. 2011;29:91-98.
20. Iqbal J, Ginsburg OM, Wijeratne TD, et al. Endometrial cancer and venous thromboembolism in women under age 50 who take tamoxifen for prevention of breast cancer: a systematic review. Cancer Treat Rev. 2012;38:318-328.

21. Smith LL, Brown K, Carthew P, et al. Chemoprevention of breast cancer by tamoxifen: risks and opportunities. Crit Rev Toxicol. 2000;30: 571-594.

22. Kaplan CP, Kim SE, Wong ST, Sawaya GF, Walsh JM, Pérez-Stable EJ. Willingness to use tamoxifen to prevent breast cancer among diverse women. Breast Cancer Res Treat. 2012;133:357-366.

23. Lin JH, Zhang SM, Manson JE. Predicting adherence to tamoxifen for breast cancer adjuvant therapy and prevention. Cancer Prev Res. 2011;4:1360-1365.

24. Armstrong K, Quistberg DA, Micco E, et al. Prescription of tamoxifen for breast cancer prevention by primary care physicians. Arch Intern Med. 2006;166:2260-2265.

25. Vogel VG, Costantino JP, Wickerham DL, et al. Update of the National Surgical Adjuvant Breast and Bowel Project Study of Tamoxifen and Raloxifene (STAR) P-2 trial: preventing breast cancer. Cancer Prev Res (Phila). 2010;3:696-706.

26. Visvanathan K, Hurley P, Bantug E, et al. Use of pharmacologic interventions for breast cancer risk reduction: American Society of Clinical Oncology clinical practice guideline. J Clin Oncol. 2013;31: 2942-2962.

27. Goss PE, Ingle JN, Ales-Martinez JE, et al. Exemestane for breastcancer prevention in postmenopausal women. N Engl J Med. 2011;364: 2381-2391.

28. Van de Velde CJ, Rea D, Seynaeve C, et al. Adjuvant tamoxifen and exemestane in early breast cancer (TEAM): a randomized phase 3 trial. Lancet. 2011;377:321-331.

29. Lazzeroni M, Serrano D, Dunn BK, et al. Oral low dose and topical tamoxifen for breast cancer prevention: modern approaches for an old drug. Breast Cancer Res. 2012;14:214.

30. Decensi A, Bonanni B, Guerrieri-Gonzaga A, et al. Biologic activity of tamoxifen at low doses in healthy women. J Natl Cancer Inst. 1998;90: 1461-1467.

31. Decensi A, Robertson C, Guerrieri-Gonzaga A, et al. Randomized double-blind $2 \times 2$ trial of low-dose tamoxifen and fenretinide for breast cancer prevention in high-risk premenopausal women. J Clin Oncol. 2009;27:3749-3756.

32. Alkner S, Bendahl P-O, Ferno M, et al. Tamoxifen reduces the risk of contralateral breast cancer in premenopausal women: results from a controlled randomised trial. Eur J Cancer. 2009;45:2496-2502.

33. Rouanet P, Linares-Cruz G, Dravet F, et al. Neoadjuvant percutaneous 4-hydroxytamoxifen decreases breast tumoral cell proliferation: a prospective controlled randomized study comparing three doses of 4-hydroxytamoxifen gel to oral tamoxifen. J Clin Oncol. 2005;23:2980-2987.
Breast Cancer: Targets and Therapy

\section{Publish your work in this journal}

Breast Cancer: Targets and Therapy is an international, peerreviewed open access journal focusing on breast cancer research, identification of therapeutic targets and the optimal use of preventative and integrated treatment interventions to achieve improved outcomes, enhanced survival and quality of life for the cancer patient.

\section{Dovepress}

View the full aims and scopes of this journal here. The manuscript management system is completely online and includes a very quick and fair peer-review system, which is all easy to use. Visit http:// www.dovepress.com/testimonials.php to read real quotes from published authors. 